\title{
Les Religions Représentent-ELles un RisQue POUR Les Droits de L'homme en France?
}

\author{
Germana Aguiar Ribeiro do Nascimento* \\ Maria José Añón Roig**
}

Introduction. 1 Les origines du principe de laïcité en France. 2 La Laïcité, mesure des libertés religieuses. Conclusion. Réferences.

\section{RESUME}

Le 13 novembre 2015 la France a été frappée par le terrorisme au nom d'un supposé idéal religieux. De ce fait une question se pose : les religions représenteraient-elles un danger pour les droits de l'homme en France? Pour mieux comprendre la conjoncture actuelle il est nécessaire de réfléchir à la relation particulière qu'a entretenue la France avec les faits religieux tout au long de l'histoire. Il est possible de vérifier que L'Etat de droit a voulu encadrer les religions pour limiter les menaces possibles à travers le principe de laïcité. Ce principe est le plus cohérent pour la garantie des droits de l'homme dans un Etat démocratique. Cependant, à force de vouloir protéger les droits de l'Homme d'une possible menace religieuse, la France a adopté un modèle de laïcité qui peut mettre en danger les libertés religieuses. Il s'agira donc d'étudier, premièrement, l'encadrement des religions qui a été réalisé au nom des droits de l'Homme au cours de l'histoire, pour après analyser la laïcité comme mesure des religions. Pour cela, la méthodologie qualitative sera utilisée. La recherche documentaire, l'étude de la jurisprudence et de la législation permettront de regrouper les renseignements relatifs au sujet.

Mots-clefs : Religions. Laïcité. Droits de l'homme. France.

* Bacharela em Direito, pela Universidade Estadual da Paraiba. Bacharela em Antropologia, pela Universidade Federal de Campina Grande. Mestre em Direito Internacional dos Direitos Humanos, pela Universidade Católica de Lyon - França. Doutoranda em Direitos Humanos, Democracia e Justiça Internacional, pela Universidade de Valência - Espanha. E-mail: germana_aguiar@hotmail.fr

** Professora efetiva de Filosofia do Direito da Universitat de València - Espanha. Licenciada e Doutora em Direito pela Universitat de València - Espanha. Diretora do Instituto Universitario de Derechos Humanos (2005-2010). Secretaria Geral da Universitat de València (20102016). Ex-diretora do Anuario de Filosofía del Derecho e atual diretora da Teoría y derecho. Revista de pensamiento jurídico. E-mail: marraja@uv.es 


\section{INTRODUCTION}

La réalité en Europe et dans le monde démontrent que les religions sont au cour de l'histoire des pays. En effet, il est difficile de trouver des groupes humains sans religion. Selon Corm ${ }^{1}$, ce besoin de religion et de transcendance est issu de la peur devant la mort et de la perplexité devant les mystères du monde. En raison de cela, depuis le XIXème siècle, plusieurs auteurs ont consacré et consacrent encore leurs temps à analyser les phénomènes religieux, ce sont des sociologues, anthropologues et philosophes qui visent à réfléchir sur le sens de la religion dans les sociétés, tels que Durkheim, Mauss et plus récemment Baubérot.

Selon Amnesty International ${ }^{2}$ la France comptait en 2008 environ 65 millions d'habitants. Il est impossible de savoir les appartenances ou opinions religieuses des citoyens, puisque aucune mention sur cela ne peut être faite au niveau de l'état civil, des recensements ou des fichiers de la fonction publique. C'est ainsi qu'il faut utiliser le sondage d'opinion pour dresser un bilan de l'état des croyances religieuses dans le pays. Le Rapport Machelon ${ }^{3}$ nous permet d'évaluer les relations des cultes avec les pouvoirs publics. Il établit le panorama du fait religieux en France. Il démontre que $65 \%$ des français se définissent comme catholiques, $6 \%$ de la population s'affirme musulmane, et que les protestants et les juifs pèsent respectivement, $2 \%$ et $1 \%$ de la population. Les $25 \%$ restants sont sans religion.

Amnesty International ${ }^{4}$ ajoute que tout Etat européen est confronté au problème de ses relations avec les religions. En France, cette situation est corroborée par la législation existante qui vise à gérer les faits religieux sur son territoire, et par plusieurs débats qui soulèvent des doutes par rapport à la liberté religieuse, à la laïcité et aux droits de l'Homme. Il est possible d'illustrer ce fait par les dernières discussions sur le port du foulard et de la burqa. Il est donc impératif de réfléchir sur la question.

Durkheim $^{5}$ a défini le terme religion comme étant "un système solidaire de croyances et de pratiques relatives à des choses sacrées, c'est-à-dire séparées, interdites, croyances et pratiques qui unissent en une même communauté morale, appelée Église, tous ceux qui y adhèrent". La religion est un phénomène collectif, plusieurs éléments sont donc communs à cette collectivité, les rites, les livres, les prescriptions morales etc. A cette définition on peut ajouter celle du dictionnaire Larousse ${ }^{6}$ qui affirme que le terme religion vient du latin religio, ce qui attache ou qui retient, et correspond à l'ensemble de croyances et de dogmes définissant le rapport de l'homme avec le sacré.

La loi française, quant à elle, ne définit pas ce qu'est une religion. La Constitution de 1958 s'y réfère seulement pour affirmer le principe de non-discrimination. En effet, respectueux du principe de laïcité, le législateur s'est toujours refusé à définir les notions de secte et de religion? ${ }^{7}$. Il sera ainsi préférable d'utiliser le terme tel qu'il est défini par le dictionnaire, sachant qu'en France, les sectes ne sont pas considérées comme religion. 
La Constitution de 1958 est le texte qui dispose de la plus forte valeur juridique dans ce pays ${ }^{8}$, elle consacre les droits de l'homme et libertés fondamentales en son sein, comme des principes de la République. En effet, toute société démocratique doit avoir comme fondement ces droits et libertés. Comme l'affirme Oberdoff', la démocratie n'existe que si elle pratique la reconnaissance, la mise en ouvre et le respect de ces droits. La Constitution dispose, de plus, son attachement à la Déclaration française de 1789 , ce qui corrobore l'importance des droits de l'Homme et des libertés fondamentales dans le pays. Les droits de l'Homme et les libertés fondamentales sont des droits liés à la nature même de l'homme, ce sont des valeurs reconnues universellement, indissociables de la dignité humaine.

De ce fait, la législation française garantit certains droits et libertés relatifs à la religion pour protéger la liberté religieuse, mais aussi pour réprimer les menaces qu'elle peut engendrer pour l'Etat de droit. Mais qu'est-ce qu'une menace? Selon le dictionnaire Larousse ${ }^{10}$ le terme vient du latin minae, et correspond à toute parole, geste, acte par lequel on exprime la volonté qu'on a de faire du mal à quelqu'un, par lequel on manifeste sa colère. Cela peut être aussi tout signe, indice qui laisse prévoir un danger.

Il est possible donc de se demander dans quelle mesure les religions constituent-elles une menace pour les droits de l'homme en France. L'Etat de droit a voulu encadrer les religions pour limiter les menaces possibles, mais en cela il a exagéré. En effet, à force de vouloir protéger les droits de l'homme de la menace religieuse, on en vient à menacer les libertés religieuses au nom des droits de l'homme. Il s'agira donc d'étudier, premièrement, l'encadrement des religions qui a été réalisé au nom des droits de l'homme au cours de l'histoire, pour après analyser la laïcité comme mesure des religions. Pour cela, la méthodologie qualitative sera utilisée. La recherche documentaire, l'étude de la jurisprudence et de la législation permettront de regrouper les renseignements relatifs au sujet.

\section{LES ORIGINES DU PRINCIPE DE LAICITE EN FRANCE}

La France est connue comme la fille aînée de l'Eglise. En effet, la religion catholique est au cour de plusieurs faits historiques dans le pays. Des violations des droits de l'Homme ont été commises au nom des religions. A partir du XIème siècle, la persécution des hérétiques emploie les méthodes les plus atroces, notamment avec l'instauration de l'Inquisition. Les hérétiques sont poursuivis par le pouvoir royal, qui cherche à les convertir au catholicisme, à confisquer leurs biens et à les emprisonner. Le droit à une justice équitable est ici oublié. De plus, l'histoire témoigne de l'utilisation d'engins de guerres pour tuer les hérétiques, ce qui dénote de l'utilisation de la torture, pratique contraire à la dignité humaine.

A compter de 1517 il est possible de vérifier un long siècle de guerres à cause des religions où les violences les plus cruelles ont été perpétrées, des droits de l'Homme étant bafoués. En effet, Martin Luther publie ses 95 thèses contes- 
tant certaines pratiques de l'Eglise catholique, telle que l'indulgence ${ }^{11}$. Cette nouvelle foi est diffusée en France par Jean Calvin, disciple de Martin Luther, qui rencontrera un fort succès, notamment dans le sud-ouest du pays.

Avec la rupture de l'unité de l'Eglise catholique, celle-ci va mettre en place un processus dans le but de récupérer le monopole de la religion. De ce fait, plusieurs guerres vont ravager le royaume de France. Dans ces guerres s'opposeront d'un côté les protestants et de l'autre, les catholiques. Il s'agit d'un contexte général d'intolérance, qui amènera à une série de huit conflits sanglants. C'est sous François Ier que débutent les premières émeutes, puisqu'il considérait que la Reforme était un obstacle à son royaume. A cet égard, Hasquin ${ }^{12}$ affirma que "ce n'est pas un hasard si le terme de tolérance au sens d'indulgence en matière de liberté religieuse fit son apparition au XVIème siècle".

Dans chaque côté, des actes féroces sont commis. Les historiens précisent que si dans un champ, les huguenots étaient décapités par Montluc, dans l'autre, les catholiques étaient obligés de se jeter d'une tour par le baron des Adrets ${ }^{13}$. Ce sont huit guerres sanglantes, la plus connue étant le massacre de la Saint-Barthélemy, le quatrième conflit qui a lieu vers 1570 . Il n'y a donc pas de respect du droit à la vie, à la liberté individuelle et à la sûreté. sécurité

En 1570, le roi Charles IX commence à écouter les conseils d'un chef protestant, l'amiral de Coligny, ce qui crée des troubles avec sa mère Catherine de Médicis, proche des catholiques. Charles IX signe un traité de paix, assurant aux protestants des places de sûreté,( ?) ce qui intensifie les querelles entre les deux camps. L'amiral de Coligny survit à un attentat commis par des catholiques, les protestants demandent donc au roi de faire justice. Le roi promet cela, ce qui maximise le climat de conflit. Le mariage entre Henri de Navarre et Marguerite, sœur du roi, qui consisterait à rétablir la paix entre protestants et catholiques ${ }^{14}$, génère au contraire plus de conflits. C'est ainsi qu'un massacre est organisé, faisant plus de 3000 victimes seulement à Paris et des milliers de morts dans d'autres villes. Le droit à la vie, à l'égalité et à la non-discrimination sont ainsi bafoués. Les protestants ne jouissent pas de la liberté d'aller et venir ni de la liberté de conscience. Il va falloir attendre plus de vingt ans pour qu'un Edit de paix durable soit instauré.

Après plusieurs Edits inefficaces instaurés durant les conflits entre catholiques et protestants, Henri IV va publier l'Edit de Nantes, un Edit de tolérance en 1598. En effet, Henry IV était lui-même un protestant et s'est converti au catholicisme pour devenir roi, ce qui dénotait une certaine sensibilité à la cause protestante. L'Edit considère donc la Réforme comme la seconde religion du Royaume, en les (lui ?) accordant un culte public ${ }^{15}$. Il prône la liberté de conscience dans son article VI, ce qui constitue une avancée pour les huguenots et l'égalité civile des protestants avec les catholiques. Il est possible de confirmer cette égalité par le fait par exemple, qu'à partir de l'Edit de Nantes les huguenots vont pouvoir vendre, acheter, léguer comme les catholiques. Les protestants pourront également accéder à tous les emplois publics et ont droit à des tribunaux spécialisés. De plus, toute violence à leur égard devient illégale. 
L'Edit va durer plus de quatre-vingt ans, instaurant un Etat catholique où les protestants sont tolérés, permettant ainsi la coexistence des deux religions. Il constitue une avancée en vue d'établir la paix entre catholiques et protestants ${ }^{16}$. Cependant, les droits des protestants restent insignifiants face aux droits des catholiques. C'est ainsi que les protestants, en tant que minorité, vont engendrer des nouveaux conflits.

Louis XIV, le roi soleil, règne de 1654 à 1715 . Il va établir une nouvelle politique en vue de reconvertir la France au catholicisme et d'uniformiser le royaume ${ }^{17}$. A partir de 1660 cette politique anti-huguenote semble commencer, plusieurs temples huguenots sont détruits, le synode national est supprimé, des dénonciations et des enquêtes sont pratiquées. Elle a eu pour conséquence d'affaiblir fortement les protestants de France et de favoriser la promulgation de l'Edit de Fontainebleau.

Le nouvel Edit révoque l'Edit de Nantes. Il compte douze articles qui sont complètement défavorables aux protestants. En effet, l'article 2 interdit tout exercice de la religion des protestants; l'article premier détermine (prévoit ?) la démolition de tout temple protestant; l'article 7 interdit les écoles protestantes; les articles 5 et 6 imposent la conversion de tous les protestants y compris les pasteurs, sous peine de bannissement, entre autres.

Au vu de cela, plus de 200000 protestants partent en exil, soit 1\% de la population française ${ }^{18}$. Il s'agit ici d'énormes violations commises au nom de la cause catholique, où des droits fondamentaux sont bafoués. C'est à cette époque que le mot réfugié apparaît dans la langue française, pour définir justement les protestants qui étaient obligés de quitter la France à cause des persécutions ${ }^{19}$. La dignité des protestants est donc méprisée. Ils sont persécutés, étant obligés soit de se convertir soit partir en exile. Louis XIV instaure les dragonnades, des missions de soldats, les dragons, qui avaient comme objectif de convertir les protestants et mettre fin à l'hérésie de Calvin. Les dragons s'installaient chez les protestants, bafouant complètement le droit à la vie privée, les obligeant à changer de religion et à donner chaque jour de grandes sommes d'argent. S'ils refusaient, ils étaient battus, subissant plusieurs formes de supplices, même devant leurs femmes et leurs enfants ${ }^{20}$.

Dorénavant, la religion des protestants était appelée religion prétendue réformée. Les protestants devraient se soumettre aux règles établies par le roi sous peine d'être envoyés aux galères. Plusieurs d'entre eux vont organiser un culte officieux, ce qui va engendrer des poursuites de l'Etat. Pour Louis $\mathrm{XIV}$, les protestants étaient un danger à la monarchie absolue, il fallait donc les supprimer.

C'est seulement à la fin du XVIIIème siècle que cette situation d'intolérance change, avec la Révolution française et la Déclaration des droits de l'homme et du citoyen, de 1789, qui représentent le début d'un processus à la fin duquel les religions sont soumises au principe de laïcité. 
En effet, après des siècles de déchirements religieux et de luttes politiques, des violations massives des droits de l'homme, la Révolution française va marquer le départ de la sécularisation de la société. Elle est considérée par certains auteurs, tels que Baubérot et Milot $^{21}$, comme le premier seuil de laïcisation. C'est ainsi un fait historique qui instaure une nouvelle vision du monde, définie dans la Déclaration des droits de l'homme et du citoyen de 1789.

La Déclaration affirme dans son article III que le principe de toute souveraineté réside dans la nation. Elle met donc fin au principe de l'autorité de droit divin, qui existait auparavant, établissant un principe de pouvoir plus rationnel. De plus, la Déclaration annule le catholicisme comme religion d'Etat, désormais, il n'y a plus de religion d'Etat, toutes les religions sont égales. Les citoyens sont égaux, protestants, juifs et catholiques peuvent être électeurs et éligibles ${ }^{22}$.

La Déclaration reconnaît aussi la liberté de conscience, l'article premier affirme "Les hommes naissent et demeurent libres et égaux en droit. Les distinctions sociales ne peuvent être fondées que sur l'utilité commune". L'article 10 affirme, quant à lui, "Nul ne doit être inquiété pour ses opinions, mêmes religieuses, pourvu que leur manifestation ne trouble pas l'ordre public établi par la loi". Il ne s'agit plus d'une simple tolérance aux protestants, mais d'une reconnaissance de l'égalité entre les citoyens et du respect de leurs croyances. A partir de là va être aussi affirmée la laïcisation des actes civils, avec la Constitution de 1791 et la laïcisation de l'Ecole. Cependant, l'influence de l'Eglise catholique ne s'arrête pas.

En effet, l'Eglise n'est pas satisfaite des changements de la Révolution. C'est ainsi que Napoleon va amorcer une pacification avec le Concordat, en 1802, à travers lequel l'Eglise accepte certains de ces changements. Toutefois, les ultra-catholiques sont contre ce concordat, ainsi que les ultra-radicaux. Il est possible de voir l'existence de deux France. Les ultra-catholiques essayent de restaurer le pouvoir de l'Eglise. Comme l'affirme Dusseau ${ }^{23}$, cette offensive est corroborée par le Syllabus de Pie IX. Il s'agit d'une compilation de toutes les idées considérées dangereuses contre lesquelles l'Eglise s'engage à lutter. Ce catalogue incluait la République comme étant une menace à l'Eglise, ainsi que tous les droits libéraux.

De plus, l'Eglise acquiert, tout au long du XIXème siècle une influence croissante dans l'enseignement. Elle veut imposer un nouveau Concordat qui lui soit plus favorable, ainsi que la restitution des status de l'état civil. Face à cela, de nouvelles Ecoles de pensée apparaissent, telles que le Positivisme, menant des idées anticléricales, fondées sur la science et la raison. Des critiques émergent, demandant la séparation de l'Eglise et l'Etat, c'est le cas de Lamennais et Benjamin Constant. C'est ainsi qui sera posé le principe de séparation entre l'Eglise et l'Etat.

Les discussions sur le projet de loi de la séparation ont durée toute l'année 1905. La loi est promulguée le 9 décembre 1905, cette loi aspire à régir l'ensemble des territoires français, mais il y a des exceptions, c'est le cas de l'Alsace-Moselle. 
Le principe de séparation entre l'Eglise et l'Etat est finalement posé, pacifiant les conflits qui ont été présents durant des décennies ${ }^{24}$.

Selon Soppelsa ${ }^{25}$, ce principe va protéger l'ensemble des institutions publiques des influences confessionnelles, il y aura donc une indépendance de l'Etat à l'égard des religions et des religions à l'égard de l'Etat. La loi 1905 pose les fondements du principe de laïcité. Toutefois, c'est seulement avec la Constitution de 1946 qu'il est affirmé comme tel, dans son article premier et dans le préambule, ce qui est confirmé après par la Constitution de 1958. Le principe se pose sur deux devoirs, qui affirment l'égalité des citoyens et la non discrimination.

Comme l'affirme Robert ${ }^{26}$, la loi interdit à l'Etat de professer une foi particulière en son nom. En effet, il est indépendant de toute foi. L'article 2 de la loi affirme ainsi que "La République ne reconnaît, ne salarie ni ne subventionne aucun culte". Elle met donc les citoyens sur un même niveau, garantissant l'égalité morale de chacun. Il ne s'agit pas d'ignorer l'existence des religions en son territoire, mais d'effacer la notion de religion reconnue par l'Etat. En effet, l'Eglise catholique était auparavant une religion reconnue, ce qui créait une distinction, discriminant les autres cultes.

Robert $^{27}$ ajoute que la loi 1905 ne souhaite pas que l'Etat entretienne des mauvaises relations avec les religions, la séparation ne représente pas une envie d'hostilité, mais tout simplement, de démontrer que le fait religieux n'est pas un fait public et pour cela, l'Etat ne peut pas subventionner ni salarier aucun culte. Il y a ainsi une obligation de l'Etat d'être neutre, de respecter toutes les religions, il ne peut pas faire la propagande d'un culte en particulier ou déclarer un culte officiel. Il ne peut pas non plus vouloir imposer une religion à ses citoyens, la liberté de conscience est donc indissociable de ce principe. Ce qui est corroboré aussi par la première partie de l'article premier de la Constitution de 1958 qui affirme que "La France est une République indivisible, laïque, démocratique et sociale".

L'Etat français est ainsi pluraliste, il doit traiter toutes les religions également, ainsi que les citoyens, indépendamment de leur appartenance religieuse. La loi 1905 vient comme réponse à toutes les persécutions qui avaient lieu en France auparavant. Comme l'affirment Baubérot et $\mathrm{Milot}^{28}$

il s'agissait d'identifier la cause principale des persécutions et des affrontements sociaux continus où les religions étaient impliquées, et d'imaginer une solution pour mettre fin aux effets délétères sur le vivre-ensemble que ces tourments occasionnent - qui vont de l'assimilation à l'élimination de ceux qui ne croient pas comme l'impose la religion dominante.

La séparation est la solution pour ces conflits. C'est ainsi que l'article premier de la Constitution de 1958, dans sa deuxième partie, affirme que "La France assure l'égalité devant la loi de tous les citoyens sans distinction d'origine, de race ou de religion. Elle respecte toutes les croyances". 
Ces auteurs confirment le devoir de tolérance de l'Etat français en montrant qu'il doit être libre pour élaborer des normes collectives dans l'intérêt général. Il doit donc être impartial et ne peut pas imposer des normes aux citoyens basées sur des critères moraux d'une religion spécifique. Il doit y avoir une autonomie de l'Etat, pour qu'aucune religion ne soit privilégiée et que les citoyens aient leurs libertés assurées. Sans cette indépendance un régime de tolérance est impossible. L'Etat est représentant de l'ensemble de la population et doit ainsi garantir le respect de la diversité et des droits de l'Homme. La neutralité de l'Etat est par conséquent obligatoire pour les services publics. Les fondements posés par la loi 1905 sont donc essentiels pour comprendre les droits et limites des libertés religieuses en France.

\section{LA LAICITE, MESURE DES LIBERTES RELIGIEUSES}

L'affirmation de la séparation entre l'Eglise et l'Etat va de pair avec l'affirmation du principe de la liberté de conscience. Briand ${ }^{29}$ affirmait en 1905 que "Non seulement la République ne saurait opprimer les consciences ou gêner, dans ses formes multiples, l'expression extérieure du sentiment religieux, mais encore $[. .$.$] elle entend respecter et faire respecter la liberté de conscience et la$ liberté des cultes".

L’article premier de la loi 1905 dispose que "La République assure la liberté de conscience. Elle garantit le libre exercice des cultes sous les seules restrictions édictées ci-après dans l'intérêt de l'ordre public". Les droits de l'Homme vont donc être la mesure des libertés religieuses. Ainsi, ils ont une fonction pacificatrice, puisqu'ils encadrent les libertés religieuses, pour qu'elles ne menacent pas l'ordre public. Cependant, il est possible de vérifier aujourd'hui, qu'au nom de ces droits de l'homme, le principe de laïcité est élargi, devenant un principe intrusif, qui porte atteinte aux libertés religieuses.

Au-delà de l'article 10 de la Déclaration de 1789, la Convention européenne des droits de l'homme précise dans son article 9 que "toute personne a droit à la liberté de pensée, de conscience et de religion ; ce droit implique (...) la liberté de manifester sa religion (...)". Le Pacte international relatif aux droits civils et politiques de 1966, à son tour, confirme la liberté de conscience, de religion et de culte. La liberté religieuse est protégée et reconnue dans l'Etat français, et correspond à deux libertés, celle de croire et celle d'exercer un culte .

La liberté de croire, selon Oberdorff ${ }^{30}$, est très voisine de la liberté d'opinion, sauf qu'elle concerne une opinion d'ordre religieuse. La loi 1905 oblige l'Etat à assurer cette liberté, imposant des sanctions dans son article 31 à ceux qui la menacent. Evans ${ }^{31}$ affirme que la liberté de croire correspond à ce qui est appelé de forum internum, c'est-à-dire la sphère de la conviction intime de l'individu, qui est inviolable. Il est impossible de séparer la liberté de conscience de la liberté de manifester cette conscience. Cependant, la liberté de croire ne donne pas à l'individu la possibilité d'agir comme il l'entend. Il peut y avoir des limites, imposées, par exemple, dans les services publics. 
Selon Lochak ${ }^{32}$ "la liberté de conscience est assimilée au for intérieur, au siège des convictions personnelles, morales et éthiques de l'individu". Malgré cela, il est difficile de faire une distinction entre liberté de conscience et la liberté d'exprimer cette conscience. En effet, l'auteur affirme q'une autonomie seulement du for interne ne saurait satisfaire les exigences de la conscience. La formulation des textes de la Déclaration de 1789 et de la Convention européenne supra mentionnés laissent entendre cela. Dans le même sens, l'article 18 de la Déclaration universelle des droits de l'homme "Toute personne a droit à la liberté de pensée, de conscience et de religion; ce droit implique la liberté de manifester sa religion (...)".

Toutefois, il est possible de vérifier que la liberté de conscience abandonne le caractère absolu quand elle est extériorisée. En effet, des limites sont imposées par l'Etat au nom de l'ordre public. C'est ainsi que les individus ne peuvent pas exprimer leurs convictions comme ils veulent, ils doivent se soumettre aux conditions de la vie en société. En France, la manifestation de la croyance est limitée dans les services publics.

Le principe de laïcité exige que les services publics soient neutres. En 2007, le Premier ministre français a publié la Charte de la laïcité dans les services publics qui rappelle aux usagers et aux agents du service public le cadre juridique qui doit être respecté, dans le but d'assurer l'application du principe de laïcité.

La Charte affirme le principe de l'égalité de tous les usagers devant le service public, une fois de plus, est garanti le principe de non discrimination. Il est encore affirmé que les usagers accueillis à temps complet dans un service public ont droit au respect de leurs croyances. Elle affirme encore que tous les usagers des services publics ont le droit d'exprimer leurs convictions religieuses dans les limites du respect de la neutralité du service. Il y a donc des limites aux droits qui doivent être respectées. C'est ainsi que le Conseil d'Etat ${ }^{33}$ en 2001 a validé la transfusion d'office d'un malade qui refusait, en tant que témoin de Jéhovah, l'administration des produits sanguins alors que cela était indispensable à sa survie.

La Charte affirme que tout agent public a un devoir de stricte neutralité, il est ainsi interdit de manifester sa conviction religieuse dans l'exercice de sa fonction. Ce qui est corroboré par l'arrêtdu Conseil d'Etat de 2009 qui condamne un guichetier de la Poste pour avoir remis aux usagers des imprimés à caractère religieux ${ }^{34}$. Dans le même sens, l'arrêt de 2003 qui condamne l'agent pour avoir utilisé les moyens de communication du service au profit d'un mouvement sectaire $^{35}$.

La liberté de conscience est aussi garantie aux agents publics, ils peuvent bénéficier d'autorisation d'absence pour participer à une fête religieuse dès lors qu'elles sont compatibles avec les nécessités du fonctionnement normal du service. A partir du principe de laïcité, plusieurs lois vont être établies visant à garantir l'ordre public et le respect des droits de l'Homme. 
L'article premier de la loi 1905 garantit aussi le libre exercice des cultes. En effet, une République laïque doit respecter tous les cultes existants dans son territoire, sans n'en privilégier aucun. Comme l'affirme Schwartz" "la laïcité française est juridiquement égalitaire et vaut pour tous les cultes qui s'expriment”. Avant la loi 1905 existait un service public du culte, organisé autour de quatre cultes: le culte catholique, le culte réformé et de la confession d'Augsburg et le culte Israélite. A partir de la loi 1905, il y a une privatisation des cultes, qui deviennent indépendants de l'Etat. Toutefois, l'Etat peut s'immiscer dans l'exercice du culte au nom de la démocratie.

Avant 1905 l'Etat assurait le fonctionnement du service public de culte à travers les ressources publiques. Après cette loi, les concours financiers ont pris fin. Schwartz ${ }^{37}$ affirme que les personnes publiques ne pouvaient plus financer le fonctionnement des cultes ni la construction d'édifices cultuels. Elles ne pouvaient pas non plus prendre en charge des frais liés à l'exercice des fonctions de ministre de culte. L'autonomie des cultes émane ainsi de la fin des concours financiers. Selon l'article 4 de la loi 1905:

Dans le délai d'un an (...) les biens mobiliers et immobiliers des menses, fabriques, conseils presbytéraux, consistoires et autres établissements publics du culte seront (...) transférés par les représentants légaux de ces établissements aux associations (...).

De ce fait, les cultes pourront s'organiser librement et déterminer leurs propres règles de vie en commun. Ils auront une personnalité juridique, comme associations cultuelles ou toute autre forme d'association, et vont disposer de leur patrimoine.

La liberté de culte, au-delà de correspondre au droit d'une communauté spécifique de se réunir et pratiquer librement et publiquement le culte de leur choix, selon leurs croyances religieuses, correspond aussi, comme l'affirme Touvet $^{38}$ à la liberté de transmettre sa foi. Cependant, ces droits sont limités au nom de l'ordre public.

Le droit à l'exercice du culte est limité s'il menace l'ordre public. Par conséquent, certains mouvements religieux peuvent se voir refuser le statut d'association cultuelle ou de congrégation, c'est le cas des sectes. En effet, l'Assemblée nationale française a publié en 2008, un rapport qui affirme qu'il est impossible de reconnaître comme religion un certain nombre de sectes qui soit ne poursuivent pas un but exclusivement religieux, soit ont des pratiques contraires à l'ordre public et aux droits et libertés d'autrui. L'administration doit ainsi évaluer s'il convient de donner le statut d'association cultuelle ou de congrégation à un certain mouvement. Selon le rapport, il existe environ 172 organisations en France à caractère sectaire qui sont dangereuses pour les droits de l'homme.

En vue de réfléchir sur les mouvements sectaires il est créé en 1998 la Mission interministérielle de lutte contre les sectes, remplacée en 2002 par la 
Mission interministérielle de vigilance et de lutte contre les dérives sectaires. En 2001, le gouvernement français publia la loi du 12 juin 2001 qui vise à prévenir et réprimer les sectes portant atteinte aux droits de l'homme et libertés fondamentales. Il est ainsi constaté que les libertés religieuses sont garanties par la loi et des limites sont établies afin de garantir la protection des droits de l'homme. Cependant, au nom de la laïcité et des droits de l'Homme, des obstacles peuvent être imposés aux libertés religieuses d'une façon exagérée et abusive.

Le principe de laïcité a été proclamé en France en vue d'établir une société pluraliste et tolérante. Le système juridique français a établi des règles pour que les libertés religieuses soient respectées. Cependant, le principe de laïcité commence, depuis une quinzaine d'années à être mal-interprété, ce qui engendre une confusion. Comme l'affirme Barbier"39, "La multitude des études qui sont consacrées à la laïcité conduit, en fait, à brouiller cette notion au lieu de la clarifier".

Il s'agit ici d'un monstre doux, d'une laïcité falsifiée, instrumentalisée pour stigmatiser une partie de la population ${ }^{40}$. Ce monstre menace les libertés religieuses, ce qui est corroboré à partir des années 80 avec l'affaire du foulard islamique. Il est préférable d'utiliser le terme foulard au terme voile. En effet, ce deuxième a une connotation péjorative, de voilement de la femme, de soumis$\operatorname{sion}^{41}$. Le contexte politique de "menace " des religions nous permet de mieux comprendre l'arrivée de cette loi.

Dans les années 70, arrivent en France des débats relativement nouveaux sur la génération des enfants de premiers immigrés qui commencent à naître. Une peur s'installe, face à des gens dont on croit qu'ils ne partagent pas le mode de vie français. A cela s'oppose la logique de la tolérance et du refus du racisme. Plusieurs événements extérieurs ont été utilisés pour alimenter ces peurs, comme la révolution islamique d'Iran et la chute du mur de Berlin, l'utopie socialiste va être remplacée par l'idéal de la république ${ }^{42}$.

En 1989, l'ayatollah Khomeyni lance une fatwa, condamnation à mort, contre l'écrivain Salman Rushdie, pour la publication des Versets sataniques. Cette action est ressentie comme une atteinte à la liberté d'expression, qui ne fait que renforcer, de ce fait, le sentiment consensuel de la défense des valeurs républicaines, en pleine année du bicentenaire de la Révolution française. En effet, l'intégrisme religieux, "courant politique radical, minoritaire et religieux se développant à la périphérie de toute religion ${ }^{43}$ ", va représenter une menace à la République. Les intégristes veulent imposer leur religion à toute la société, étant ainsi contre les valeurs démocratiques, et surtout contre l'Etat laïque. Ces personnes ont une logique de haine et de fanatisme. C'est ainsi qu'ils peuvent, au nom de Dieu, commettre des actes terroristes, comme l'a fait Mohamed Merah en 2012, à Toulouse et plus récemment, en 2015, les frères Kouachi et Abdeslam, à Paris.

Cependant, l'intégrisme religieux n'existe pas seulement au sein des islamistes mais aussi parmi les catholiques. C'est ainsi que des catholiques radicaux du groupe Renouveau Français ${ }^{44}$ ont interrompu violemment une pièce 
de Romeo Castellucci en 2011, au nom de leur religion. Toutefois, la menace islamique est plus ressentie, puisque mélangée avec la difficulté des français de digérer la présence de la population islamiste en son territoire. L'intégrisme religieux présente une menace, il heurte davantage la culture française quand il est attaché à l'Islam. Il y a donc un amalgame à travers lequel tous les musulmans sont vus comme une menace à la République. Le terme Islamophobie apparaît dans la fin des années 1980 pour désigner justement cette peur irraisonnée de l'islam. Des symptômes de cet amalgame sont remarqués dans l'affaire de 1989 .

L'humoriste Coluche, cité par Baubérot et Milot ${ }^{45}$ illustre bien l'ambivalence du débat par sa fameuse phrase "Le racisme c'est comme les arabes ça ne devrait pas exister". Il est donc possible de voir que l'expulsion des filles du lycée en 1989 pour avoir refusé d'enlever leur foulard pour un cours de sport et de technologie et les conséquentes décisions n'arrivent pas sans coïncidence, c'est une déformation du principe de laïcité qui menace les libertés religieuses.

Après 1989, les débats sur l'utilisation des signes religieux ont continué en France. Le Conseil d'Etat et la Circulaire Bayrou ont donné leurs avis sur l'expulsion des trois filles. C'est ainsi qu'en 2003, une Commission est nommée pour rédiger un rapport sur l'application du principe de laïcité. Il s'agit de la Commission Stasi, qui publia son rapport le 11 décembre 2003, portant une forte influence à la publication de la loi 2004. Il a été très critiqué par plusieurs auteurs, tels que Gresh ${ }^{46}$, puisqu'il prône le respect de l'égalité entre homme et femme pour défendre l'interdiction du port du foulard, sans prendre en considération l'opinion des sociologues ni des femmes concernées. Depuis, cette loi n’a pas apaisé les tensions, bien au contraire. Il a été donc nécessaire pour les politiciens de promulguer la loi de 2010.

Face à la menace représentée par l'utilisation du foulard, le Conseil d'Etat ${ }^{47}$ va donner son avis en 1989. Il va affirmer que:

dans les établissements scolaires, le port par les élèves de signes par lesquels il entendent manifester leur appartenance à une religion n'est pas par lui-même incompatible avec le principe de laïcité, dans la mesure où il constitue l'exercice de la liberté d'expression et de manifestation de croyances religieuses, mais que cette liberté ne saurait permettre aux élèves d'arborer des signes d'appartenance religieuse qui, par leur nature,(...) ou par leur caractère ostentatoire ou revendicatif, constitueraient un acte (...) qui troublerait l'ordre.

Ainsi, le port par lui-même des signes religieux n'est pas en soi une menace au principe de laïcité. Cependant, la liberté d'expression des croyances religieuses est assortie des limites, comme l'affirme Pollet-Panoussis ${ }^{48}$ : elle ne peut pas troubler l'ordre public.

On voit donc que le fait d'affirmer que le port en soi d'un signe religieux ne porte pas atteinte à la laïcité dénote de la tolérance du Conseil de l'Etat. 
Cependant, le fait de donner la capacité aux établissements et à ses autorités pour définir les modalités d'application et de sanction rend possible des dérives irrespectueuses des libertés religieuses. C'est le cas en 1992, quand des exclusions sont prononcées en raison de l'utilisation des signes religieux qui étaient interdits d'une façon générale et absolue dans le règlement intérieur d'un établissement. Le Conseil d'Etat va donc annuler les décisions d'exclusion ${ }^{49}$. Au-delà de l'avis du Conseil d'Etat, une circulaire va compliquer la question.

La circulaire Bayrou est publiée le 20 septembre 1994, établissant une distinction entre les signes discrets et les signes ostentatoires. Selon la circulaire, en respect au principe de laïcité, il est nécessaire d'interdire les signes "si ostentatoires que leur signification est précisément de séparer certains élèves des règles de vie commune de l'école".

Différemment de l'avis du Conseil d'Etat, la circulaire "passe du comportement ostentatoire, au signe qui serait en lui-même, ostentatoire ${ }^{50}$ ". De plus, malgré le fait que le Conseil d'Etat ait déjà abordé le sujet, cette circulaire apporte une vision plus précise qui vise non pas à interdire tout signe ostentatoire, mais surtout le foulard. C'est ce qui est interprété par les autorités des établissements scolaires.

Un fait qui peut corroborer cela c'est l'affaire Ait Ahmad ${ }^{51}$. Dans cette affaire, une jeune élève a été renvoyée de son établissement scolaire parce qu'elle ne voulait pas enlever son foulard pour un cours de sport et de technologie. Le règlement intérieur de l'établissement interdisait "le port de signes religieux ostentatoires et constitutifs de prosélytisme ou discrimination". Le Conseil d'Etat a validé la décision du dit établissement, confirmant la décision d'exclusion du Collège.

Cette décision dénote une certaine confusion dans l'application de la loi. Le concept de discrétion est difficile à définir dans un texte, ainsi que le concept de signe. Dans un lieu de savoir et d'apprentissage, il est contradictoire d'interdire un signe sans en expliquer le contexte. De plus, dans quelle mesure le port d'un signe porterait-il atteinte à la sécurité ou à l'ordre ? C'est plutôt la prétention d'une envie d'ordre qui porte atteinte aux libertés des élèves, et cela va continuer avec la publication de la loi de 2004 et celle de 2010.

La loi publiée le 15 mars 2004 interdit "le port de signes ou de tenues par lesquels les élèves manifestent ostensiblement une appartenance religieuse dans les écoles, lycées et collèges publics". C'est ainsi qui sont insérés dans le Code de l'Education les articles L-141-5 et L-141-5-1 qui ajoutent que la mise en œuvre d'une mesure disciplinaire doit être précédée par le dialogue. Il s'agit dorénavant d'une loi, qu'au nom de l'application du principe de laïcité, promeut la discrimination et l'intolérance.

Amnesty Internationa ${ }^{52}$ manifestait sa crainte à l'égard des conséquences négatives de cette loi. Elle évoque ensuite les problèmes que la loi a engendré après trois ans de sa publication. A la rentrée scolaire 2004/2005, 47 élèves avaient été 
exclus, c'est le cas de Singh . Le Conseil d'Etat ${ }^{53}$ a affirmé:

considérant qu'en estimant que le sous-turban porté par Ranjit A. dans l'enceinte scolaire, bien qu'il soit d'une dimension plus modeste que le turban traditionnel et de couleur sombre, ne pouvait être qualifié de signe discret et que l'intéressé, par le seul port de ce signe, a manifesté ostensiblement son appartenance à la religion sikhe la cour administrative d'appel de Paris n'a pas fait une inexacte application des dispositions de l'article L. 141-5-1 du Code de l'éducation.

Au-delà des exclusions, certains élèves ont abandonné leurs études ; d'autres sont partis à l'étranger. D'autres se sont inscrits au Centre National d'Enseignement à distance et dans les établissements catholiques, qui les permet d'utiliser leur signe.

Il est donc possible de confirmer que la crainte d'Amnesty International s'est concrétisée. Cependant, la décision du Comité des droits de l'homme porte un peu d'espoir. En effet, après avoir perdu dans le tribunal administratif de Paris, dans la Cour administrative d'appel et finalement dans le Conseil d'Etat, Singh a saisi le Comité des droits de l'homme en 2008. Le Comité ${ }^{54}$ condamne la France et affirme que

la France n'a pas fourni d'élément probant pour affirmer qu'en portant son turban, Singh porterait atteinte aux droits et libertés des autres élèves ou à l'ordre public à l'école (...) le renvoi définitif de l'école publique a constitué une punition disproportionnée, qui a eu de graves effets sur l'éducation à laquelle il aurait dû avoir droit en France, comme toute personne de son âge.

L'avis du Comité demande à la France une indemnisation et le changement de la loi 2004.

Avant cette décision, la France a publié une autre loi qui porte aussi atteinte aux droits de l'homme. Elle porte sur l'utilisation de signes religieux qui dissimulent le visage dans l'espace public. La loi a été votée, même si les professeurs de droit consultés à l'époque de la Mission parlementaire d'information ont affirmé que "le port du voile intégral dans l'espace public n'est pas en soi, une atteinte au principe de laïcité55". La mission préfère utiliser et divulguer les arguments qui sont pour la loi, alors que les arguments contre la loi sont nombreux ${ }^{56}$. Baubérot et Milot ${ }^{57}$ citent Ducomte, qui s'interroge sur "l'aptitude de la société française à digérer certaines situations" et Fourest, qui affirme que l'application de cette loi fait la France aller "vers une laïcité autoritaire qui produirait plus d'effets pervers que d'effets positifs". Les arguments pour la loi, quant à eux, visent d'une façon explicite la religion musulmane. Quillardet affirmait que "la vision d'une burqa dans la rue porte atteinte à ma propre liberté de conscience ${ }^{58}$ ".

La loi est publiée le 11 octobre 2010 et interdit ainsi la dissimulation du visage dans l'espace public, sa conformité avec la Constitution française étant 
affirmée par le Conseil constitutionnel ${ }^{59}$. La loi est suivie de deux circulaires en 2011, où l'on prévoit l'amende pour ceux qui dissimulent le visage dans l'espace public, soit $150 €$, et que cela correspond à une contravention de 2ème classe.

Ainsi que les loi précédentes, cette loi a été très critiquée. Le Commissaire aux droits de l'homme du Conseil de l'Europe ${ }^{60}$, Hammarberg, l'affirmait en 2010 "L'interdiction générale du voile intégral serait une mesure bien mal inspirée, portant atteinte à la vie privée. Toute loi d'interdiction pourrait aussi poser de sérieux problèmes de compatibilité avec la Convention européenne des droits de l'homme". Un rapport sur les libertés religieuses produit aux Etats-Unis en 2012 va dans le même sens, l'ambassadrice affirme que porter ou non un signe religieux doit être un choix personnel ${ }^{61}$.

Malgré ces critiques, la Cour européenne des droits de l'homme a validé l'interdiction du voile intégral dans l'affaire S.A.S c. France ${ }^{62}$, du 1 juillet 2014. La Cour souligne que

la préservation des conditions du « vivre ensemble » était un objectif légitime à la restriction contestée et que notamment au regard de l'ample marge d'appréciation dont la France disposait sur cette question de politique générale suscitant de profondes divergences, l'interdiction posée par la loi du 11 octobre 2010 n'était donc pas contraire à la Convention des Droits de l'Homme.

Dans le même sens, dans un arrêt du 26 novembre 2015, l'affaire Ebrahimian c. France ${ }^{63}$, la Cour affirme que le non-renouvellement du contrat, dans un établissement public, d'une assistante sociale refusant d'ôter son voile n'est pas une violation à la Convention européenne des droits de l'homme. Elle souligne que

les autorités nationales n'ont pas outrepassé leur marge d'appréciation en constatant l'absence de conciliation possible entre les convictions religieuses de Mme Ebrahimian et l'obligation de s'abstenir de les manifester, ainsi qu'en décidant de faire primer l'exigence de neutralité et d'impartialité de l'État.

Ces décisions ont aussi généré des débats. Izza Leghtas ${ }^{64}$ (2015) affirme que des lois comme celles en vigueur en France et en Belgique "portent atteinte aux droits des femmes qui choisissent de porter le voile, et font en même temps peu pour protéger celles qui sont forcées de le faire". Corella ${ }^{65}$ souligna que ces décisions démontrent le problème de la discrimination et renforcent l'inégalité. Pierre Bourdieu ${ }^{66}$ affirmait déjà lors des débats sur le port des voiles à l'école que

en projetant sur cet événement mineur, d'ailleurs aussitôt oublié, le voile des grands principes, liberté, laïcité, libération de la femme, etc., les éternels prétendants au titre de maître à pensér ont livré, comme dans un texte projectif, leurs prises de position inavouées sur le problème de l'immigration : du fait que la question patente - faut-il ou non accepter à l'école le port du voile dit islamique? occulte la question latente - faut-il ou non accepter en France les 
immigrés d'origine nord-africaine ? - ils peuvent donner à cetter dernière une réponse autrement inavouable.

Il est donc visible qu'il y a une interprétation du principe de laïcité qui est erronée et qui menace les libertés religieuses. Cela démontre que la laïcité est en crise, puisqu'en réalité elle exige que chaque personne puisse pratiquer ses idées religieuses sans privilèges ou obstacles ${ }^{67}$, ce qu'est impossible en France. Comme l'affirma Javier de Lucas $^{68}$, on ne peut pas parler de securité si celle-ci imposa des limites exagérées aux libertés et aux droits de certains groupes. Cet auteur ajouta que l'égalité est un principe reconnu et garanti dans les constitutions et dans l'Union Européenne et doit ainsi être respecté.

\section{CONCLUSION}

Après des siècles de conflits, le principe de séparation entre l'Eglise et l'Etat est finalement posé le 9 décembre 1905 en France. La République est désormais laïque et doit respecter tous les cultes existants sur son territoire, sans en privilégier aucun. Le principe de laïcité est donc proclamé en vue d'établir une société pluraliste et respectueuse des droits de l'Homme. Ce principe est le plus cohérent pour la garantie des droits de l'homme dans un Etat démocratique.

Cependant, dans les années 70, arrivent en France des débats relativement nouveaux sur l'Islam. En effet, les fils de la vague des premiers immigrés commencent à naître. Une peur s'installe, face à des gens dont on croit qui ne partagent pas le mode de vie français. Les attentas terroristes ne font qu'augmenter cette peur. C'est ainsi qu'au nom de la laïcité, des lois sont publiées créant des obstacles aux libertés religieuses d'une façon exagérée et abusive. Tout cela au nom de la laïcité, des droits de l'homme et de la securité. Malgré le fait que le principe de laïcité soit le plus cohérent pour une société démocratique, l'on verifie qu'il peut être défiguré, ne faisant qu'augmenter la discrimination, l'exclusion et l'inégalité. De plus, l'Etat d'urgence installé dans le pays après les derniers attentats peut aussi être une menace aux libertés au titre de la sécurité.

Si le discours de la tolérance a été important dans l'histoire, aujourd'hui il ne peut plus être accepté. Il ne faut plus demander à l'Etat et à la société que telle religion soit tolérée, il est impératif que toutes les religions soient respectées d'une manière égale. Le modèle de laïcité « à la française » doit ainsi être évalué puisqu'il ne garantit pas l'égalité. Seulement ainsi nous pourrons parler d'un Etat démocratique et républican.

\section{REFERENCES}

ALAIN, Gest; GUYARD, Jacques. Les sectes en France. Paris: Assemblée Nationale, 2008.

AMNESTY INTERNATIONAL. Droit et religions dans les Etats membres de l’Union européenne. Paris : Amnesty International, 2011. 
AYCARD, Mathilde; REMOND, René. Atlas de l'Histoire de France. Paris: France Loisirs, 1996.

BARBIER, Maurice. Pour une définition de la laïcité française. Paris: Le Débat, n. 134, 2005.

BAUBÉROT, Jean. La laïcité falsifiée. Paris: La Découverte, 2012.

BAUBÉROT, Jean; ZUBER, Valentine. Une haine oubliée. L'antiprotestantisme avant le "pacte laïque "(1870-1905). Paris: Albin Michel, 2000.

BAUBÉROT, Jean; MILOT Micheline. Laïcités sans frontières. Paris: Editions du Seuil, 2011.

BORELLO, Céline. Le secret dans la lutte contre l'hérésie en Provence. Rives nord-méditerranéennes, Marseille, 2004.

BOURDIEU, Pierre. Interventions, 1961-2001: science sociales et action politique. Marseille: Agonne, 2002.

BRIAND, Aristide. Député. La Séparation des Eglises et de l'Etat. Paris: ARD Cornély et Cie, 1905.

CARBONNIER-BURKARD, Marianne; CABANEL, Patrick. Une histoire des protestants en France. Paris: Desclée de Brouwer, 1998.

CERF, Martine ; HORWITZ, Marc ; DELFAU, Gérard. Dictionnaire de la laïcité. Paris: Armand Colin, 2011.

CONSEIL CONSTITUTIONNEL. Décision du 7 octobre 2010, Loi interdisant la dissimulation du visage dans l'espace public, requête numéro 2010-613 DC.

CONSEIL D’ETAT. Décision du 27 novembre 1989, M. Kherouaa, requête numéro 346893. méro 130394.

. Décision du 2 novembre 1992, M. Kherouaa et autres, requête nu-

. Décision du 20 octobre 1999, M. et Mme. Ait Ahmad, requête numéro 181486.

198546.

. Décision du 26 octobre 2001, Mme. Senanayake, requête numéro 244428.

. Décision du 15 octobre 2003, M. Jean-Philippe O, requête numéro

. Décision du 5 décembre 2007, M. Singh, requête numéro 285394.

. Décision du 19 février 2009, M. Bouvier, requête numéro 311633.

CORELLA, Angeles Solanes. Límites a los derechos en el espacio público: mujeres, velos y convivencia. Valencia: Cuadernos Electrónicos de Filosofía del Derecho, n 31, 2015. <https://www.academia.edu/13099387/\%C3\%ADmites_a_los_de- 
rechos_en_el_espacio_público_mujeres_velos_y_convivencia_Limiting_rights_ in_public_space_women_veils_and_conviviality_>Accès le 02 décembre 2015.

CORM, Georges. La question religieuse au XXIème siècle. Paris: La Découverte, 2007.

COUR EUROPEENNE DES DROITS DE L'HOMME. Affaire communiquée du 1er juillet 2014, S.A.S. c./ France, requête numéro 43835/11.

. Décision du 26 novembre 2015, Ebrahimian c. France, requête numéro 64846/11.

DE LUCAS, Javier. ¡Igualdad, no tolerancia!. Al revés y al derecho, Valencia, 2015. <http://alrevesyalderecho.infolibre.es/?p=3541>. Accès le 15 nov. 2015

DEBRAY, Régis. Le " fait religieux " : définitions et problèmes. Portail national des professionnels de l'éducation, Paris, 2002. <http://eduscol.education. $\mathrm{fr} /$ cid46334/le-fait-religieux-\%A0-definitions-et-problemes.html> . Accès le 5 février 2014.

DICTIONNAIRE LAROUSSE. <http://www.larousse.fr> Accès le 15 novembre 2015

DURKHEIM, Emile. Les formes élémentaires de la vie religieuse. Paris: PUF, 1914.

DUSSEAU, Joëlle. L'histoire de la séparation, entre permanences et ruptures. La laïcité ou la « religion » de la République. Revue politique et parlementaire, Paris, n. 1038, 2006.

EVANS, Malcolm. Manuel sur le port des symboles religieux dans les lieux publics. Strasbourg : Editions du Conseil de l'Europe, 2009.

FEILLET, Bernard; ESCADA, Alain. Des catholiques intégristes s'attaquent au Theâtre. Les Observateurs, Paris, 2011. <http://observers.france24.com/fr/ content/20111028-extreme-droite-catholique-civitas-castellucci-blaspheme-theatre-ville-paris-integriste-christ>. Accès le 13 février 2015.

GONZALEZ-PACHECO, Antonio. La Revolución Francesa (1789-1799). Barcelona: Ariel Practicum, 1998.

GRESH, Alain. Les faux semblants de la Commission Stasi. Islam \& Laïcité, Paris, 2004. <http://www.islamlaicite.org/article187.html>. Accès le 02 février 2013.

GUEANT, Claude. Direction des libertés publiques et des affaires juridiques. Ministère de l'Intérieur. Laïcité et liberté religieuse. Recueil de textes et de jurisprudence, Paris, Edition des Journaux officiels, 2011.

HASQUIN, Hervé (org.). Histoire de la laïcité. Bruxelles : La Renaissance du livre, 1979.

HUMAN RIGHTS COMMITTEE. Communication du 1 novembre 2012, Singh v. France, numéro 1852/2008. 
JOUANNA, Arlette. La Saint-Barthélemy - les mystères d'un crime d'Etat. Paris: Gallimard, 2007.

La CEDH valide l'interdiction du voile en France. AriegeNews, Ariège, 2015. <http://www.ariegenews.com/ariege/actualites_economie/2014/78300/la-cedh-tranche-sur-l-interdiction-du-voile-integral-en-france.html>. Accès le 13 novembre 2015.

LOCHAK, Daniel. For intérieur et liberté de conscience. CURAPP, Paris, 1995. <https://www.u-picardie.fr/curapp-revues/root/35/daniele_lochak. pdf_4a081ea8c78af/daniele_lochak.pdf>. - Accès le 4 février 2015.

MACHELON, Jean-Pierre. Rapport Machelon. Ministére de l'Intérieur et de l'aménagement du territoire, Paris, $2006<$ http://www.ladocumentationfrancaise.fr/rapports-publics/064000727/index.shtml\#book_sommaire>. -Accès le 02 février 2015.

MAILLOUX, Louise. L'intégrisme religieux menace-il nos droits ? Colloque Égalité et Laïcité, quelles perspectives? Montréal et Québec, 2010. <http:// louisemailloux.wordpress.com/2011/01/16/lintegrisme-religieux-menace-t-il-nos-droits/> - Accès le 13 février 2015.

MONDONICO-TORRI, Cécile. Les réfugiés en France sous la monarchie de juillet : l'impossible statut. Revue d'histoire moderne et contemporaine, Paris, n. 47, 2000.

OBERDORFF, Henri. Droits de l'homme et libertés fondamentales. Paris: L.G.D.J, Lextenso éditions, 2010.

POLLET-PANOUSSIS, Delphine, Le point de vue du juge administratif. Manifester sa religion: droits et limites, Paris, L'Harmattan, 2011.

QUENIART, Jean. La Révocation de l'Edit de Nantes. Paris: Desclée de Brouwer, 1985.

Que dit la loi? Mission Interministérielle de vigilance et de lutte contre les dérives. Paris, 2015. <http://www.derives-sectes.gouv.fr/quest-ce-quune-dérive-sectaire/que-dit-la-loi> Accès le 02 février 2015.

ROBERT, Jacques. Les fondements juridiques de la laïcité. Revue politique et parlementaire, Paris, n 1038, 2006.

RUIZ MIGUEL, Alfonso ; NAVARRO VALLS, Rafael. Laicismo y Constitución. Madrid, Fundación Coloquio Jurídico Europeo, 2008.

SENIGUER, Haoues. La laïcité à l'épreuve de l'islam et des musulmans : le cas de la France. Revue d'éthique et de théologie morale, Paris, n 254, 2009.

SOPPELSA, Jacques. De la laïcité. Revue politique et parlementaire, Paris, $\mathrm{n}$ 1038, 2006.

SCHWARTZ, Rémy. Un siècle de laïcité. Paris: Berger-Levrault, 2007. 
Washington critique les lois anti-burqa en France et en Belgique. Le point, Paris, 2012. <http://www.lepoint.fr/societe/washington-critique-les-lois-anti-burqa-en-france-et-en-belgique-30-07-2012-1491116_23.php>. Accès le 3 février 2014.

TOUVET, Laurent. La liberté de culte en droit français. Actes du colloque international organisé par le Conseil de la communauté marocaine à l' étranger, Fès, 2009. <http://www.ccme.org.ma/fr/Evénements-du-CCME/Colloque-internationalStatut-juridique-de-l-Islam/la-liberte-de-culte-en-droit-francais-laurent-touvet-france.html>. Accès le 04 février 2014.

1 CORM, Georges. La question religieuse au XXIème siècle. Paris : La Découverte, 2007, p. 81.

2 AMNESTY INTERNATIONAL. Droit et religions dans les Etats membres de l'Union européenne, Paris, 2011, p. 71.

3 MACHELON, Jean-Pierre. Rapport Machelon. Ministére de l'Intérieur et de l'aménagement du territoire, Paris, $2006<$ http://www.ladocumentationfrancaise.fr/rapports-publics/064000727/index. shtml\#book_sommaire>. Accès le 02 février 2015.

4 AMNESTY INTERNATIONAL. Droit et religions dans les Etats membres de l'Union européenne, Paris, 2011, p. 71.

5 DURKHEIM, Emile. Les formes élémentaires de la vie religieuse. Paris : PUF, 1914, p. 60.

6 Dictionnaire Larousse. <http://www.larousse.fr/dictionnaires/francais/religion/67904? q=religion\#67154>. Accès le 02 novembre 2015.

7 Que dit la loi? Mission Interministérielle de vigilance et de lutte contre les dérives, Paris, 2015. <http:// www.derives-sectes.gouv.fr/quest-ce-quune-dérive-sectaire/que-dit-la-loi> - Accès le 02 février 2015.

8 OBERDORFF, Henri. Droits de l'homme et libertés fondamentales. Paris : L.G.D.J, Lextenso éditions, 2010, p. 146.

9 Idem, p. 147.

10 Dictionnaire Larousse. <http://www.larousse.fr/dictionnaires/francais/menace/50414? q=menace\#50305>. Accès le 02 novembre 2015.

11 HASQUIN, Hervé (org.). Histoire de la laïcité. Bruxelles :La Renaissance du livre, 1979, p. 35.

12 Idem, p. 37.

13 AYCARD, Mathilde; REMOND, René. Atlas de l'Histoire de France. Paris, France Loisirs, 1996, p. 126.

14 JOUANNA, Arlette. La Saint-Barthélemy: les mystères d'un crime d'Etat. Gallimard, Paris, 2007, p. 10.

15 BORELLO, Céline. Le secret dans la lutte contre l'« hérésie » en Provence. Rives nord-méditerranéennes, Marseille, 2004, p. 107.

16 QUENIART, Jean. La Révocation de l'Edit de Nantes. Desclée de Brouwer, Paris, 1985, p. 13.

17 BAUBÉROT, Jean ; ZUBER, Valentine. Une haine oubliée. L'antiprotestantisme avant le " pacte laïque " (1870-1905). Paris, Albin Michel, 2000, p. 5.

18 AYCARD, Mathilde; REMOND, René, op. cit. , p. 144.

19 MONDONICO-TORRI, Cécile. Les réfugiés en France sous la monarchie de juillet : l'impossible statut. Revue d'histoire moderne et contemporaine, Paris, n 47, 2000, p. 732.

20 CARBONNIER-BURKARD, Marianne; CABANEL, Patrick. Une histoire des protestants en France. Paris, Desclée de Brouwer, 1998, p. 27.

21 BAUBÉROT, Jean; MILOT Micheline. Laïcités sans frontières. Paris : Editions du Seuil, 2011, p. 20.

22 GONZALEZ-PACHECO, Antonio. La Revolución Francesa (1789-1799). Ariel Practicum, Barcelona, 1998, p. 45.

23 DUSSEAU, Joëlle. L'histoire de la séparation, entre permanences et ruptures. La laïcité ou la " religion " de la République. Revue politique et parlementaire, Paris, n 1038, 2006, p. 15.

24 CERF, Martine ; HORWITZ, Marc ; DELFAU, Gérard. Dictionnaire de la laïcité. Armand Colin, Paris, 2011, p. 227.

25 SOPPELSA, Jacques. De la laïcité. Revue politique et parlementaire, Paris, n 1038, 2006, p. 2.

26 ROBERT, Jacques. Les fondements juridiques de la laïcité. Revue politique et parlementaire, Paris, n 1038, 2006, p. 9.

27 Idem, p. 10. 
28 BAUBÉROT, Jean ; MILOT Micheline, op. cit., p. 76.

29 BRIAND, Aristide. Député. La Séparation des Eglises et de l’Etat. ARD Cornély et Cie, Paris, 1905.

30 OBERDORFF, Henri, op. cit., p. 445.

31 EVANS, Malcolm. Manuel sur le port des symboles religieux dans les lieux publics. Strasbourg : Editions du Conseil de l'Europe, 2009, p. 8.

32 LOCHAK, Daniel. For intérieur et liberté de conscience. CURAPP, Paris, 1995. <https://www.u-picardie. $\mathrm{fr} /$ curapp-revues/root/35/daniele_lochak.pdf_4a081ea8c78af/daniele_lochak.pdf>. - Accès le 4 février 2015.

33 CONSEIL D'ETAT. Décision du 26 octobre 2001, Mme. Senanayake, requête numéro 198546.

34 CONSEIL D’ETAT. Décision du 19 février 2009, M. Bouvier, requête numéro 311633.

35 CONSEIL D’ETAT. Décision du 15 octobre 2003, M. Jean-Philippe O., requête numéro 244428.

36 SCHWARTZ, Rémy. Un siècle de laïcité. Paris, Berger-Levrault, 2007, p. 159.

37 Idem, p. 159.

38 TOUVET, Laurent. La liberté de culte en droit français. Actes du colloque international organisé par le Conseil de la communauté marocaine à l' étranger, Fès, 2009. <http://www.ccme.org.ma/fr/Evénements-du-CCME/Colloque-internationalStatut-juridique-de-l-Islam/la-liberte-de-culte-en-droit-francaislaurent-touvet-france.html>. Accès le 04 février 2014.

39 BARBIER, Maurice. Pour une définition de la laïcité française. Le Débat, Paris, n 134, 2005, p. 130.

40 BAUBÉROT, Jean. La laïcité falsifiée. Paris, La Découverte, 2012, p. 8.

41 SENIGUER, Haoues. La laïcité à l'épreuve de l'islam et des musulmans : le cas de la France. Revue d'éthique et de théologie morale, Paris, n 254, 2009, p. 86.

42 Idem, p. 87.

43 MAILLOUX, Louise. L'intégrisme religieux menace-il nos droits? Colloque Égalité et Laïcité, quelles perspectives? Montréal et Québec, 2010. <http://louisemailloux.wordpress.com/2011/01/16/lintegrismereligieux-menace-t-il-nos-droits/>. Accès le 13 février 2015.

44 FEILLET, Bernard; ESCADA, Alain. Des catholiques intégristes s'attaquent au Theâtre. Les Observateurs, Paris, 2011. <http://observers.france24.com/fr/content/20111028-extreme-droite-catholique-civitas-castellucci-blaspheme-theatre-ville-paris-integriste-christ>. - Accès le 13 février 2015.

45 BAUBÉROT; MILOT, op. cit. , p. 291.

46 GRESH, Alain. Les faux semblants de la Commission Stasi. Islam \& Laïcité, Paris, 2004. <http://www. islamlaicite.org/article187.html>. - Accès le 02 février 2013.

47 CONSEIL D’ETAT. Décision du 27 novembre 1989, M. Kherouaa, requête numéro 346893.

48 POLLET-PANOUSSIS, Delphine, Le point de vue du juge administratif. Manifester sa religion: droits et limites, Paris, L'Harmattan, 2011, p. 141.

49 CONSEIL D'ETAT. Décision du 2 novembre 1992, M. Kherouaa et autres, requête numéro 130394.

50 BAUBÉROT; MILOT, op. cit., p. 296.

51 CONSEIL D'ETAT. Décision du 20 octobre 1999, M. et Mme. Ait Ahmad, requête numéro 181486.

52 AMNESTY INTERNATIONAL, op. cit., p. 72.

53 CONSEIL D’ETAT. Décision du 5 décembre 2007, M. Singh, requête numéro 285394.

54 HUMAN RIGHTS COMMITTEE. Communication du 1 novembre 2012, Singh v. France, numéro $1852 / 2008$.

55 BAUBÉROT; MILOT, op. cit., p. 301.

56 Idem, p. 302.

57 Ibidem, p. 302.

58 SCHWARTZ, op. cit. , p. 159

59 CONSEIL CONSTITUTIONNEL. Décision du 7 octobre 2010, Loi interdisant la dissimulation du visage dans l'espace public, requête numéro 2010-613 DC.

60 Washington critique les lois anti-burqa en France et en Belgique. Le point, Paris, 2012. <http://www. lepoint.fr/societe/washington-critique-les-lois-anti-burqa-en-france-et-en-belgique-30-07-2012-1491116_23. php>. Accès le 3 février 2014.

61 Idem.

62 COUR EUROPEENNE DES DROITS DE L'HOMME. Affaire communiquée du 1er juillet 2014, S.A.S. c./ France, requête numéro 43835/11.

63 COUR EUROPEENNE DES DROITS DE L'HOMME. Décision du 26 novembre 2015, Ebrahimian c. France, requête numéro 64846/11.

$64 \mathrm{La}$ CEDH valide l'interdiction du voile en France. AriegeNews, Ariège, 2015. <http://www.ariegenews. 
com/ariege/actualites_economie/2014/78300/la-cedh-tranche-sur-l-interdiction-du-voile-integral-en-france. html>. - Accès le 13 novembre 2015.

65 CORELLA, Angeles Solanes. Límites a los derechos en el espacio público: mujeres, velos y convivencia. Cuadernos Electrónicos de Filosofía del Derecho. Valencia, n 31, 2015. <https://www.academia. edu/13099387/\%C3\%ADmites_a_los_derechos_en_el_espacio_público_mujeres_velos_y_convivencia_Limiting_rights_in_public_space_women_veils_and_conviviality_>. Accès le 02 décembre 2015.

66 BOURDIEU, Pierre. Interventions, 1961-2001 : science sociales et action politique Marseille, Agonne, 2002, p. 305.

67 RUIZ MIGUEL, Alfonso ; NAVARRO VALLS, Rafael. Laicismo y Constitución. Madrid, Fundación Coloquio Jurídico Europeo, 2008, p. 113.

68 DE LUCAS, Javier. ¡Igualdad, no tolerancia!. Al revés y al derecho. Valencia, 2015. <http://alrevesyalderecho.infolibre.es/?p=3541>. Accès le 15 novembre 2015.

\title{
DO RELIGIONS REPRESENT A RISK TO HUMAN RIGHTS IN FRANCE
}

\begin{abstract}
On the November 13th, 2015, France was hit by a terrorist attack in the name of a supposed religious ideal. Thus one question arises: would religions represent a threat to human rights in France? For a better understanding of the current situation it is necessary to consider the special relationship France has maintained with the religious facts throughout history. It is possible to verify that the constitutional state wanted to frame the religions through the principle of secularism to limit possible threats. Indeed, this principle is the most consistent for the guarantee of human rights in a democratic state. However, wishing to protect the human rights from a possible religious threat, France has adopted a secular model that can endanger religious freedoms. Therefore, this article will examine, first, the framing of religions that has been done in the name of human rights throughout history. After that, it will analyze the principle of secularism as a measure of religions. For this, qualitative methodology will be used. The literature, the study of the jurisprudence and the legislation will support the information relating to the subject.
\end{abstract}

Keywords: Religions. Secularism. Human Rights. France.

Submetido: 6 dez. 2015

Aprovado: 5 jan. 2016 